\title{
Unilateral absence of foramen spinosum with bilateral ophthalmic origin of the middle meningeal artery: case report and review of the literature
}

\author{
E. Cvetko ${ }^{1}$, R. Bosnjak ${ }^{2}$ \\ ${ }^{1}$ Institute of Anatomy, Faculty of Medicine, University of Ljubljana, Ljubljana, Slovenia \\ ${ }^{2}$ Department of Neurosurgery, University Medical Centre, University Hospital Centre, Ljubljana, Slovenia
}

[Received 3 July 2013; Accepted 7 August 2013]

Bilateral ophthalmic origin of the middle meningeal artery with an unilateral absence of foramen spinosum has not yet been described. We report on a skull with endocranial meningeal grooves indicating bilateral ophthalmic origin of the middle meningeal artery, however, its branches were normal both in their position and distribution. In addition, a rare venous sinus variation was present unilaterally - a sinus of Hyrtl. Imaging identification of the anomalous origin of the middle meningeal artery is important while planning surgical and endovascular interventions in the middle cranial fossa and the orbit. (Folia Morphol 2014; 73, 1: 87-91)

Key words: anomaly, foramen spinosum, middle meningeal artery, ophthalmic artery, sinus Hyrtl

\section{INTRODUCTION}

Anomalous origin and variations in the course of the medial meningeal artery (MMA) are of clinical significance. Imaging identification of the origin of the MMA is important while planning surgical and endovascular interventions in the region of the skull base. MMA was found to origin from the third part (sphenomaxillary portion) of the maxillary artery [22, $31]$, ophthalmic artery $[4,15,29,35]$, persistent stapedial artery [1], internal carotid artery [7, 27] and basilar artery $[19,30]$.

The anomalous origin of the MMA is associated with the agenesis of the foramen spinosum (FS) [25]. Royle and Motson [29] have described a case of bilateral origin of the MMA from the ophthalmic artery with bilateral absence of foramen spinosum (FS) in the adult skull. Up to our knowledge, aplasia of the conventional MMA with its origin from the ophthalmic artery in the presence of the FS of normal position and size has not been reported yet. We performed a literature search of the MEDLINE database up to the year 2012. The date of the last search was December 2012. The following keywords were queried singly and in combination: middle meningeal artery, variation, ophthalmic artery, foramen spinosum, absence, anatomy, anomaly, origin, sinus Hyrtl. The search was limited to studies published in English, German and French. We report on female cadaver found to have an unilateral absence of FS with endocranial meningeal grooves indicating bilateral ophthalmic origin of the MMA. In addition, a rare venous sinus variation was present unilaterally - a sinus of Hyrtl, an entity which has not been reported in such anatomical variation yet.

\section{CASE REPORT}

The brachycephalic skull, which had well-defined bony markings, was that of a contemporary Caucasian female (60-70 years). The bones of the skull were normal. The foramina on the base of the skull were symmetrical and within normal limits of size 


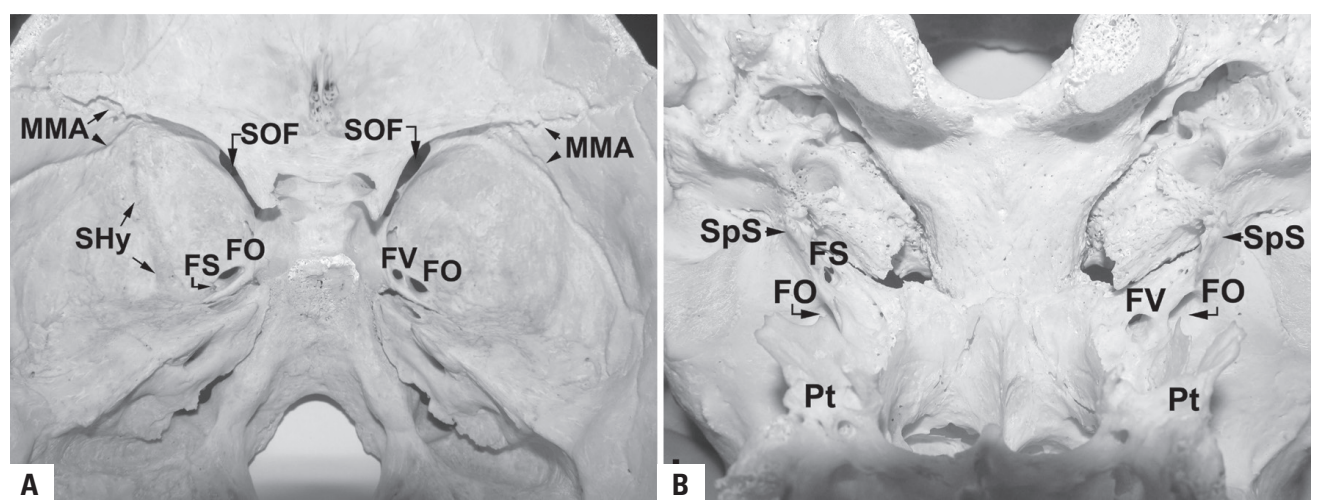

Figure 1. Superior (A) and inferior view (B) of the skull base showing the absence of the foramen spinosum on the right and foramen spinosum of normal position and size on the left. Endocranial meningeal grooves indicate the bilateral ophthalmic origin of the middle meningeal artery (MMA). On the left the groove which probably housed the sinus of Hyrtl (SHy) arose from the lateral part of the smaller wing of the sphenoid and ended at foramen spinosum (FS); FO — foramen ovale; FV — foramen of Vesalius; SOF — superior orbital fissure; $\mathrm{Pt}$ — pterygoid process; $\mathrm{SpS}$ — spina sphenoidalis.

and distribution with the following exceptions: on the right there was the absence of the FS and the presence of the foramen of Vesalius anteromedially to the foramen ovale (Figs. 1A, B). On the left the FS was of normal size and position. The long axis of the foramen ovale was $7 \mathrm{~mm}$ on both sides and of the single FS on the left $3 \mathrm{~mm}$.

The origin and distribution of the endocranial meningeal grooves were bilaterally symmetrical and indicated that the main stem of the MMA arose from the lateral limits of the superior orbital fissure (SOF) (Figs. 1A, 2A, B). On both sides the groove of the main stem of the MMA passed laterally for $25 \mathrm{~mm}$, grooving the greater wing of the sphenoid bone, and ended by dividing into anterior and posterior divisions, which were normal both in their position and distribution (Figs. 1A, 2A, B). There were no endocranial meningeal grooves of MMA in the floor of the middle cranial fossa (greater wing of sphenoid bone) on both sides (Figs. 1A, 2A, B).

On the left, an unusual anteroposterior and shallow groove was identified on the floor of the middle cranial fossa. It was approximately $6 \mathrm{~mm}$ in width and passed from the lateral part of the smaller wing of the sphenoid bone (close to the branching point of the MMA) posteromedially and ended at the FS. The groove probably housed the sinus of Hyrtl, emptying through the FS into the pterygoid plexus. No other variations or pathology were identified intracranially or extracranially.

\section{DISCUSSION}

The MMA in most individuals arises from the maxillary branch of the external carotid artery and enters the FS. It then divides into anterior and posterior branches to supply the dura and adjacent calvarium [17]. The complex embryology underlying the development of the MMA gives rise to a number of anomalous origins, courses and anastomoses involving this vessel [24]. We present a case of bilateral ophthalmic origin of the MMA with the absence of the FS on the right and a rare venous sinus anomaly - sinus of Hyrtl traveling through the single FS on the left side.

The endocranial groove housing the stem of the MMA started bilaterally from the lateral edge of the SOF, similarly to the case described by Royle and Motson [29] and Diamond [6], pointing to the ophthalmic origin of the MMA. There was no groove on the lateral bone of the orbit in the case presented, and the foramen meningo-orbitale was absent bilaterally. In case of Royle and Motson [29], foramen meningo-orbitale was missing as well. Low [22] described 1 case in which the main stem of the MMA arose in the orbit bilaterally and passed through the foramen meningo-orbitale just lateral to the SOF, the FS being absent. The groove was continued on the lateral side of the orbit to the inferior orbital fissure indicating that the origin of the MMA was from the third part of the maxillary artery.

In the case presented there was no groove starting from the foramen ovale to indicate the passage of the MMA, as in 2 cases $(n=1200)$, in which the FS was absent, as reported by Chandler and Derezinski [3]. On the basis of the intracranial meningeal grooves observed in our case we can hypothesise that the MMA was of ophthalmic origin, but we cannot specify whether the artery arose directly from the ophthalmic artery stem or from the lacrimal artery, or the groove represents an enlarged channel of otherwise normally 


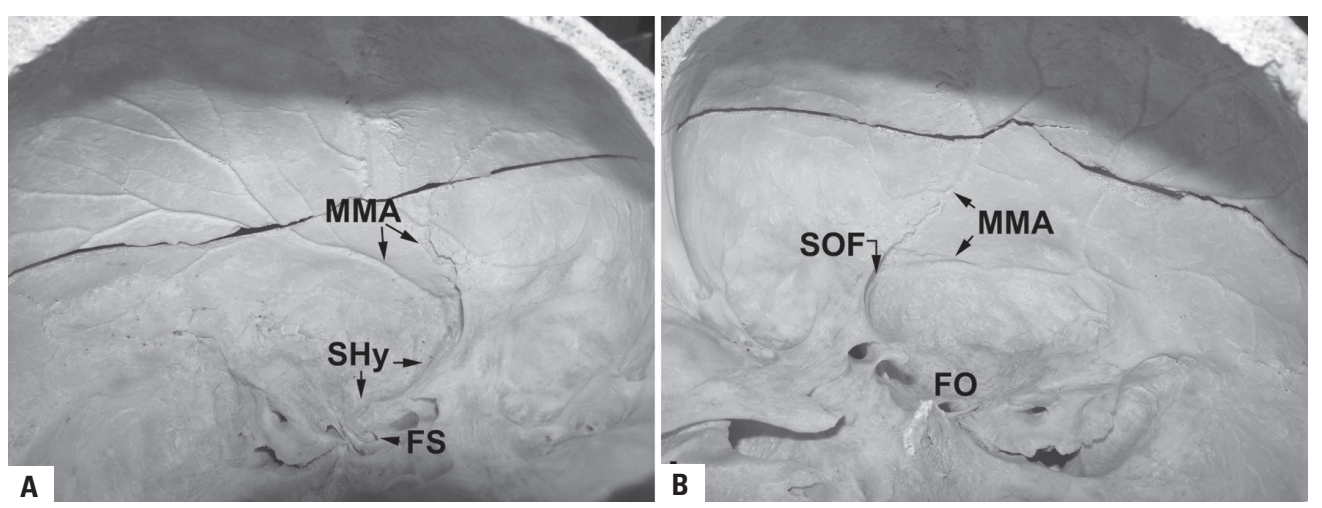

Figure 2. View of the inside of the skull (A — left, B — right) showing the grooves of the middle meningeal arteries (MMA); SOF — superior orbital fissure; FO — foramen ovale; SHy — sinus of Hyrtl; FS — foramen spinosum.

occurring anastomosis between the MMA and the lacrimal artery (sphenoidal and meningolacrimal artery).

Very few cases of the main stem of the MMA arising in the orbit have been documented. Report of Royle and Motson [29] is the first photographically documented report. Zukerkandl [35] described 4 cases in which the main stem of the MMA arose in the orbit. The MMA of the ophthalmic origin is known as the ophthalmic MMA (OMMA) [29, 35]. It passes through the lateral end of the SOF or a foramen in the greater wing of the sphenoid - foramen meningo-orbitale. Gabriele and Bell [15] consider their report of 3 cases of the ophthalmic origin of the MMA as the first arteriographic demonstration of the anomaly.

Instances of partial origin of the MMA from the ophthalmic artery (only anterior branch) have been reported as well. Toida [31] in his study of 192 Chinese skulls found 5 instances of anterior branch, and only 1 instance of the complete stem of the MMA originating from the orbit. Curnow [4] described a single case in which, on the right side only, there was a small FS through which passed a small branch of the maxillary artery supplying only the trigeminal ganglion. A large branch of the ophthalmic artery passed through the SOF and gave off all the other branches of the MMA - left side was normal. Chandler and Derezinski [3] undertook a survey of the origin and course of the MMA in 1,200 hemi-skulls and found only a single case of the anterior division and no case of the main stem of the MMA arising in the orbit. Klisovic et al. [18] found $1 \%$ incidence of the cases of partial origin of the MMA from the ophthalmic artery in the analysis of 186 skulls. Dilenge and Ascherl [7] found 17 cases of MMA arising partly or completely from the ophthalmic artery among 3,500 arteriograms (0.5\%).
Anomalous origin of the MMA accompanies the absence of the FS. Bilateral absence of the FS was rarely reported $[22,29]$. The FS was found to be unilateral in $1 \%(1 / 100$ skulls $)$ or $1 / 199(0.5 \%)$ of the foramina examined by Berge and Bergman [2]. Nikolova et al. [28] reported unilateral FS in 1.9\% $(5 / 257)$ of medieval skulls and $0 \%$ at contemporary series (200 skulls). McLennan et al. [25] reported to found only 1 skull with unilateral absence of the FS examining 108 dry skulls. Roentgenographic study of Lindblom [20] reported $0.4 \%$ and study with high resolution computed tomography by Ginsberg et al. [16] 3.2\% of the cases with absent FS (123 patients).

We could not find the report in the literature on the ophthalmic origin of the MMA in the presence of singular FS as observed on the left side in the case presented. It is believed that the ossification of the posterior border of the greater wing of the sphenoid progressively developed around the MMA during the course of human evolution. It is evident from our case that aplasia of the conventional MMA occurs in the presence of the FS, pointing that formation of FS and MMA are independent processes.

The anomalous origin of the MMA can be explained in view of the complex embryologic development of the MMA, which leads to its normal extracranial origin and intracranial distribution. The available embryological literature on the development of cranial arteries suggests a fundamental relationship between the development of the ophthalmic, MMA and stapedial artery [7]. The stapedial artery is formed by the union of the remnants of the first branchial arch with the hyoid artery. After having penetrating the ring of the stapes it divides into 2 divisions: 1) maxillofacial division consisting of an 
infraorbital and a manidbular branch; 2) supraorbital division consisting of branches destined to supply the orbit and the intracranial segment of the MMA. The branches of the maxillofacial division are assimilated by the developing external carotid artery and form the maxillary artery (including the extracranial part of the MMA). At this stage, the proximal part of the stapedial artery involutes, and its remnants become the tympanic branches of the MMA.

Two separate processes are involved in the anomaly of the ophthalmic origin of the MMA: 1) failure of the proximal intraorbital and retroorbital stapedial branches to involute, so that the intracranial segment of the MMA remains connected with the intraorbital stapedial branches; 2 ) defective involution of the maxillofacial division of the stapedial artery, so that the extracranial segment of the MMA is never formed. As a result, no connection forms between the maxillary artery and the intracranial segment of the MMA [7].

The MMA occasionally gives off the ophthalmic artery [21]; rarely, the ophthalmic artery may give off the anterior division of the MMA [18] and, more rarely, the ophthalmic artery may give off the main stem of the MMA, as in the case presented.

An unusual finding of a rare intracranial venous sinus was observed on the left side - the sinus of approximately $6 \mathrm{~mm}$ in width travelled from the groove of the sphenoparietal sinus to the FS - this sinus corresponded to the rarely reported accessory venous sinus of Hyrtl [32]. This sinus is described as beginning near the SOF (the sphenoparietal sinus) and travelling posteriorly into the transverse sinus. It may, however, leave the skull via foramen ovale or spinosum to drain into pterygoid plexus [32], as seen in the case presented.

Unusual patterns of the MMA may affect surgical and endovascular treatment of highly vascularised tumours, such as meningiomas and arterio-venous dural fistulas in the orbit and middle cranial fossa. Convexity meningiomas often receive an arterial supply from the meningeal vessels. These locations of meningiomas at the skull base may also be encountered within aberrant middle meningeal artery territory: sphenoid wing meningiomas, anterior clinoid process meningiomas, middle cranial fossa meningiomas, parasellar space meningiomas, SOF meningiomas, tuberculum sellae meningiomas, diaphragm sellae and optic canal(s) meningiomas, posterior clinoid process, dorsum sellae, upper clival meningiomas, Dorello's space meningiomas, and petroclival meningiomas [13]. Anterior clinoidectomy, either extradural [8] or intradural, is a crucial step during pterional approach to the central skull base used for clipping carotid-ophthalmic $[12,33]$, giant internal carotid artery and paraclinoid aneurysms and upper basilar artery aneurysms $[5,9]$, to gain entrance to the cavernous sinus for direct surgical management of carotid-cavernous fistulae, intracavernous aneurysms and parasellar macroadenomas, and for resection of extracavernous tumours such as craniopharyngiomas, periclinoid and suprasellar meningiomas [34], and giant pituitary adenomas. The relative increase in exposure of the optic nerve, internal carotid artery, and opticocarotid triangle by removal of the anterior clinoid process is well documented [14].

The principle of early devascularisation in meningioma microsurgery includes identification of feeders and proximal obliteration by coagulation or preoperative embolisation. Ophthalmic origin of the MMA is of surgical interest because it may influence the choice for a different surgical approach to the same lesion. Anomalous meningeal arteries originating from the ophthalmic artery may be encountered during pterional approach to the central cranial base in which the dura is first elevated from the greater and lesser wings of the sphenoid and later during dural incision of the SOF in extradural approach to cavernous sinus [13]. Ophthalmic artery and its anomalous ramifications may be identified in the orbital apex [26].

The adequate surgical exposure of the ophthalmic artery at its origin on the superomedial aspect of the intradural internal carotid artery (ICA) just behind distal dural ring is a demanding procedure that combines epidural and intradural approaches. The main feature of the epidural phase is a removal of the anterior clinoid process (extradural anterior clinoidectomy) and unroofing of the optic canal. Incision of the dura of the SOF and separating parietal dural leaf from the visceral one enables exposure of triangles of the lateral wall of cavernous sinus $[10,11]$. In the intradural trans-sylvian phase of the approach, the dura propria of the optic nerve is cut along the nerve in the unroofed canal, exposing ophthalmic artery from its outspring from ICA into the proximal optic canal on the inferiomedial aspect of the optic nerve [12]. An increased diameter of the ophthalmic artery can be expected with OMMA. A development of carotid-ophthalmic aneurysm is more likely with increased flow in OMMA. Also, middle meningeal artery aneurysms are well-known to be associated with a meningioma [23].

The increased use of superselective angiography and embolisation of tumours may result in more frequent identification of anomalous meningeal artery 
origin. Consideration should be given to the possible presence of this anomaly in examining angiographic studies done in preparation for carotid embolisation procedures or operations directed along the sphenoid ridge.

\section{ACKNOWLEDGEMENTS}

The technical assistance of Ivan Blažinovič and Friderik Štendler and the figure labeling of Marko Slak are highly acknowledged.

\section{REFERENCES}

1. Altman F (1947) Anomalies of the internal carotid artery and its branches; their embryological and comparative anatomical significance. Report of a new case of persistent stapedial artery. Laryngoscope, 57: 313-319.

2. Berge JK, Bergman RA (2001) Variations in size and in symmetry of foramina of the human skull. Clin Anat, 14: 406-413.

3. Chandler SB, Derezinski CF (1935) The variations of the middle meningeal artery within the middle cranial fossa. Anat Rec, 62: 309-319.

4. Curnow J (1873) Two instances of irregular ophthalmic and middle meningeal artery. J Anat Physiol, 8: 55-156.

5. Day JD, Giannotta SL, Fukushima T (1994) Extradural temporopolar approach to lesions of the upper basilar artery and infrachiasmatic region. J Neurosurg, 81: 230-235.

6. Diamond MK (1991) Homologies of the meningeal-orbital arteries of humans: a reappraisal. J Anat, 178: 223-241.

7. Dilenge D, Ascherl GF Jr (1980) Variations of the ophthalmic and middle meningeal arteries: relation to the embryonic stapedial artery. Am J Neuroradiol, 1: 45-54.

8. Dolenc VV (1985) A combined epi- and subdural direct approach to carotid-ophthalmic artery aneurysms. J Neurosurg, 62: 667-672.

9. Dolenc VV, Skrap M, Sustersic J, Skrbec M, Morina A (1987) A transcavernous-transsellar approach to the basilar tip aneurysms. Br J Neurosurg, 1: 251-259.

10. Dolenc VV (1989) Anatomy and surgery of the cavernous sinus. Springer Verlag, Wien.

11. Dolenc VV (1993) Direct microsurgical repair of intracavernous vascular lesions. J Neurosurg, 58: 824-831.

12. Dolenc VV (1999) A combined transorbital-transclinoid and transsylvian approach to carotid-ophthalmic aneurysms without retraction of the brain. Acta Neurochir Suppl, 72: 89-97.

13. Dolenc VV (2003) Microsurgical anatomy and surgery of the central skull base. Springer Verlag, Wien.

14. Evans JJ, Hwang YS, Lee JH (2000) Pre- versus post-anterior clinoidectomy measurements of the optic nerve, internal carotid artery, and opticocarotid triangle: a cadaveric morphometric study. Neurosurgery, 46: 1018-1023.

15. Gabriele OF, Bell D (1967) Ophthalmic origin of the middle meningeal artery. Radiology, 89: 841-844.

16. Ginsberg LE, Pruett SW, Chen MY, Elster AD (1994) Skull-base foramina of the middle cranial fossa: reassessment of normal variation with high-resolution CT. Am J Neuroradiol, 15: 283-291.

17. Hollinshead WH (1982) Anatomy for surgeons: the head and neck. $3^{\text {rd }}$ Ed. Lippincott Williams \& Wilkins, Philadelphia.

18. Klisović D, Šikić E, Krmpotić-Nemanić J (1993) Variations of the middle meningeal artery: significance for surgery and practice. Clin Anat, 6: 289-294.

19. Kumar S, Mishra NK (2012) Middle meningeal artery arising from the basilar artery: report of a case and its probable embryological mechanism. J Neurointerv Surg, 4: 43-44.

20. Lindblom K (1936) A roentgenographic study of the vascular channels of the skull with a special reference to intracranials tumors and arteriovenous aneurysms. Acta Radiol Suppl, 30: 1-146.

21. Liu Q, Rhoton AL Jr (2001) Middle meningeal origin of the ophthalmic artery. Neurosurgery, 49: 401-406.

22. Low FN (1946) An anomalous middle meningeal artery. Anat Rec, 95: 347-351.

23. Maekawa H, Tanaka M, Hadeishi H (2009) Middle meningeal artery aneurysm associated with meningioma. Acta Neurochir, 151: 1167-1168.

24. Manjunath KY (2001) Anomalous origin of the middle meningeal artery: a review. J Anat Soc India, 50: 179-183.

25. McLennan JE, Rosenbaum AE, Haughton VM (1974) Internal carotid origins of the middle meningeal artery. The ophthalmic-middle meningeal and stapedial-middle meningeal arteries. Neuroradiology, 7: 265-275.

26. Morard M, Tcherekayev V, de Tribolet N (1994) The superior orbital fissure: a microanatomical study. Neurosurgery, 35 : 1087-1093.

27. Newton TH, Potts DG (1974) Radiology of the skull and brain. In: Newton TH, Potts DG eds. Angiography (arteries). Vol. 2. The CV Mosby Co, St Louis, pp. 1261-1269.

28. Nikolova SY, Toneva DH, Yordanov YA, Lazarov NE (2012) Absence of foramen spinosum and abnormal middle meningeal artery in cranial series. Anthropol Anz, 69: 351-366.

29. Royle G, Motson R (1973) An anomalous origin of the middle meningeal artery. J Neurol Neurosurg Psychiatry, 36: 874-876.

30. Shah QA, Hurst RW (2007) Anomalous origin of the middle meningeal artery from the basilar artery: a case report. J Neuroimaging, 17: 261-263.

31. Toida N (1934) Ueber die endokranischen Furchen der arteria meningea media bei den Chinesen. J Orient Med, 21: 13-14.

32. Tubbs RS, Loukas M, Shoja MM, Oakes WJ (2007) Accessory venous sinus of Hyrtl. Folia Morphol, 66: 198-199.

33. Yasargil MG, Gasser JC, Hodosh RM, Rankin TV (1977) Carotid-ophthalmic aneurysms: direct microsurgical approach. Surg Neurol, 8: 155-165.

34. Yonekawa Y, Ogata N, Imhof H-G, Olivecrona M, Strommer K, Kwak T, Roth P, Groscurth P (1997) Selective extradural anterior clinoidectomy for supra- and parasellar processes. J Neurosurg, 87: 636-642.

35. Zuckerkandl E (1876) Zur Anatomie der Orbitalarterien. Medizinische Jahrbiicher der K.K. Gesellschaft der Arzte zu Wien, Wein. 